\title{
Crescimento e produção de genótipos de bananeiras em clima subtropical
}

\author{
Growth and production of banana genotypes in subtropical climate
}

\author{
Manoel Euzébio de Souza ${ }^{\mathrm{I}}$ Sarita Leonel ${ }^{\mathrm{II}}$ Alexandre Martins Fragoso ${ }^{\mathrm{II}}$
}

RESUMO

O presente trabalho teve como objetivo avaliar as características de crescimento e o potencial produtivo de genótipos de bananeira em Botucatu/SP. Foram avaliadas características de crescimento, tais como altura de planta, circunferência do pseudocaule, número de folhas e número de dias entre o florescimento e a colheita. Estas medidas foram realizadas na emissão da inflorescência. Foram mensuradas também as características de produção, como peso do cacho, número de frutos, peso médio dos frutos, produtividade, número de pencas; peso, número, comprimento e diâmetro dos frutos da $2^{a}$ penca. $O$ delineamento adotado foi o inteiramente casualisado, com 11 tratamentos (genótipos), cinco repetições e duas plantas úteis por parcela experimental. Os genótipos que apresentaram melhor desempenho agronômico, dentro de cada grupo genomico, foram 'Grand Naine'(AAA), 'Thap Maeo' (AAB) e 'Fhia 01' (AAAB).

Palavras-chave: Musa spp, cultivares, ciclo de produção.

ABSTRACT

The objective of this research was to evaluate the growth characteristics and the productive potential genotypes of banana tree in Botucatu/SP. It was evaluated growth characteristics such as plant height, pseudostem circumference, number of leaves, number of days between flowering and harvest, measured at the flowering time. It was also measured production characteristics, such as bunch weight, number fruits, average weight of the fruits, productivity, number of hands and weight, number, length and diameter of the $2^{\text {nd }}$ bunch. The experimental design was completely randomized with 11 treatments (genotypes), five replications and two plants per plot. The genotypes that showed the better agronomic performance within each genomic group were 'Grand Naine'(AAA), 'Thap Maeo' $(A A B)$ e 'Fhia 01' $(A A A B)$.

Key words: Musa spp, cultivars, production cycle.

\section{INTRODUÇÃO}

A banana é a fruta tropical mais degustada e a segunda mais colhida no mundo. Nutritiva, acessível à maioria da população e disponível o ano todo, é o quarto produto alimentar mais consumido no mundo. No Brasil, a banana perde apenas para laranja em volume de produção (ANUÁRIO BRASILEIRO..., 2009).

O Brasil produziu em 2008 o equivalente a 7.217.824 toneladas de banana, cultivadas em uma área

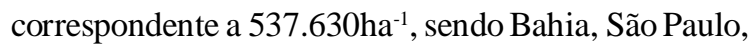
Santa Catarina, Pará, Minas Gerais, Ceará e Pernambuco os principais responsáveis pela produção nacional. São Paulo é o segundo maior produtor, com uma produção de 1.222.820 toneladas (ANUÁRIO BRASILEIRO..., 2009). Explorado em sua maioria por pequenos agricultores, o cultivo da bananeira exerce importante papel socioeconômico em muitos países emergentes, contribuindo não só para a geração de renda, mas também para a fixação da mão de obra no meio rural. Estimativas dão conta de que a cultura é responsável por mais de 500 mil empregos diretos (ANUÁRIO BRASILEIRO..., 2009).

'Departamento de Produção Vegetal, Faculdade de Ciências Agronômicas (FCA), Universidade Estadual Paulista (UNESP), 18610307, Botucatu, SP, Brasil. E-mail: manoelrural@yahoo.com.br. Autor para correspondência.

"Departamento de Produção Vegetal, FCA, UNESP, Botucatu, SP, Brasil. 
Apesar do grande número de cultivares existentes no Brasil, são poucos os que apresentam potencial agronômico com fins comerciais, principalmente considerando a preferência dos consumidores, produtividade, o porte das plantas e os aspectos biométricos superiores dos frutos. Uma das estratégias para a solução dos entraves mencionados é a seleção de novos genótipos com características superiores.

Sabendo da importância da bananicultura para o Brasil, sobretudo para o Estado de São Paulo, o presente trabalho teve como objetivo avaliar características de crescimento e produção de genótipos de bananeira em clima subtropical.

\section{MATERIAL E MÉTODOS}

O experimento foi conduzido em um bananal com três anos de implantação no pomar experimental do Departamento de Produção Vegetal - Horticultura da Faculdade de Ciências Agronômicas - UNESP, Campus de Botucatu/SP, que apresenta as coordenadas geográficas: $22^{\circ} 55^{\prime} 55^{\prime \prime} \mathrm{S}, 48^{\circ} 26^{\prime} 22^{\prime \prime} \mathrm{O}$ e altitude $810 \mathrm{~m}$. O tipo climático predominante no local é o temperado quente (mesotérmico) com chuvas no verão e seca no inverno (Cwa - Koppen), tendo temperatura média anual de $20,5^{\circ} \mathrm{C}$ e precipitação pluviométrica média anual de $1533 \mathrm{~mm}$ (CUNHA et al., 1999). O solo da área foi classificado como Nitossolo Vermelho, segundo os critérios da EMBRAPA (2006). O plantio foi realizado no mês de janeiro/2006, sendo avaliado neste trabalho o ano agrícola de 2009. O espaçamento adotado foi de $2,5 \mathrm{~m} \times 2,5 \mathrm{~m}$, e as covas foram abertas com dimensões de $60 \mathrm{~cm}$ de diâmetro por $60 \mathrm{~cm}$ de profundidade. Foram realizadas, para todos os genótipos de bananeira, adubações orgânica e mineral nas covas de plantio, além disso, foram feitas adubações de cobertura aos 30, 60, 90 e 120 dias após o plantio, conforme as recomendações técnicas para a cultura (TEIXEIRA et al., 1997). Durante o período do experimento, as plantas receberam tratos culturais, tais como: irrigação complementar, controle de plantas daninhas, que foram realizadas através de capinas manuais sempre que necessário, desbaste, que foi feito a cada dois meses a partir do surgimento dos primeiros filhotes, retirada de folhas secas quinzenalmente, eliminação do coração, quando este distanciava 10$12 \mathrm{~cm}$ da última penca e o corte do pseudocaule, realizado após a colheita.

Os genótipos avaliados foram de três grupos genômicos: (AAA) 'Nanicão-IAC-2001', 'Grand Naine', 'Caipira' e 'Nam'; (AAB) 'Thap Maeo', 'PrataAnã' e 'Prata-Zulu'; (AAAB), 'FHIA 01', 'FHIA18', 'Prata-Graúda' e 'Maçã Tropical.'

As características de crescimento avaliadas foram: altura de plantas, circunferência do pseudocaule e número de folhas, medidas na época da emissão da inflorescência. Em relação ao ciclo da planta, foi avaliado o intervalo em dias entre o florescimento e a colheita. Também foram mensuradas características de produção, tais como: peso do cacho, número de frutos, peso médio dos frutos, número de pencas, peso, número, comprimento, diâmetro de frutos da $2^{a}$ penca e a produtividade.

Foi adotado o delineamento experimental inteiramente casualisado, com 11 tratamentos (genótipos), cinco repetições e duas plantas úteis por parcela experimental, rodeadas por quatro plantas bordadura. Foi feita análise de variância e, quando houve diferença significativa, os dados foram comparados pelo teste de Tukey a 5\% de probabilidade. A comparação entre as médias foi realizada dentro de cada grupo genômico.

\section{RESULTADOS E DISCUSSÃO}

Os dados referentes ao crescimento e ao ciclo compreendido entre a emissão da inflorescência e a colheita estão apresentados na tabela 1. Os genótipos que apresentaram maior altura dentro de cada grupo genômico foram 'Grand Naine' e 'Caipira' (AAA), 'Prata Zulu' e 'Thap Maeo' (AAB), porém, para o grupo (AAAB), não houve diferença entre os genótipos.

A altura de plantas é considerada um dos principais descritores sob o ponto de vista fitotécnico e de melhoramento, pois está ligada aos aspectos de densidade de plantio, produção e manejo da cultura (GONÇALVES et al., 2008). Todavia, em um cultivar comercial, é indesejável que a bananeira expresse valores muito elevados, pois pode dificultar a colheita e provocar o tombamento da planta em decorrência de ventos fortes e ataque de nematoides e broca (LEDO et al., 1997).

LEDO et al. (2008), avaliando o desempenho agronômico de 20 genótipos de bananeira no município de Propriá-SE, constataram altura de $2,17 \mathrm{~m}$ para o cultivar 'Grand Naine' e 3,25m para 'Caipira', em um ciclo de avaliação. RAMOS et al. (2009), trabalhando com diferentes genótipos de bananeira em BotucatuSP, observaram que o cultivar 'Prata Zulu' apresentou maior porte $(3,0 \mathrm{~m})$ quando comparado com os cultivares 'Thap Maeo' e 'Prata Anã' que atingiram alturas de 2,50 e 2,10m, respectivamente. RAMOS et al. (2009) verificaram, no primeiro ciclo de produção, que o híbrido 'Maçã tropical' teve altura de 3,20m, sendo superior aos híbridos 'Fhia 01', 'Prata Graúda' e 'Fhia 18', que atingiram respectivamente alturas de 2,60, 2,70 e 2,80m.

Para a circunferência do pseudocaule, observa-se que, no grupo genômico AAA, o cultivar 'Grand Naine' apresentou maiores valores $(77,80 \mathrm{~cm})$, 
Tabela 1 - Valores médios de altura da planta, circunferência do pseudocaule, número de folhas por planta e número de dias do florescimento à colheita de acessos de bananeira, Botucatu/SP (março 2009).

\begin{tabular}{|c|c|c|c|c|}
\hline Cultivares & Altura de plantas $(\mathrm{m})$ & $\begin{array}{l}\text { Circunferência do } \\
\text { Pseudocaule }(\mathrm{cm})\end{array}$ & $\begin{array}{l}\text { Número de folhas por } \\
\text { planta }\end{array}$ & $\begin{array}{l}\mathrm{N}^{\mathrm{o}} \text { de dias do } \\
\text { Florescimento à Colheita }\end{array}$ \\
\hline & \multicolumn{4}{|c|}{ - } \\
\hline 'Grand Naine' & $3,04 \mathrm{~A}$ & $77,80 \mathrm{~A}$ & $10,80 \mathrm{~A}$ & $154,00 \mathrm{~A}$ \\
\hline 'Nanicão’ & $2,40 \mathrm{~B}$ & $71,60 \mathrm{AB}$ & $10,68 \mathrm{~A}$ & $103,00 \mathrm{C}$ \\
\hline 'Caipira' & $3,20 \mathrm{~A}$ & $68,00 \mathrm{~B}$ & $10,20 \mathrm{~A}$ & $121,00 \mathrm{~B}$ \\
\hline 'Nam' & $2,70 \mathrm{~B}$ & $64,60 \mathrm{~B}$ & $10,20 \mathrm{~A}$ & $100,00 \mathrm{C}$ \\
\hline $\mathrm{CV} \%$ & 11,06 & 6,38 & 11,79 & 7,81 \\
\hline & \multicolumn{4}{|c|}{ 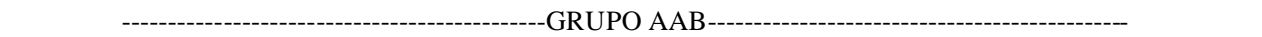 } \\
\hline 'Thap Maeo' & $3,44 \mathrm{~A}$ & $71,60 \mathrm{~B}$ & $10,30 \mathrm{~A}$ & $96,00 \mathrm{~B}$ \\
\hline 'Prata Anã' & $3,00 \mathrm{~B}$ & $81,60 \mathrm{AB}$ & $11,40 \mathrm{~A}$ & $152,00 \mathrm{~A}$ \\
\hline 'Prata Zulu’' & $3,68 \mathrm{~A}$ & $84,80 \mathrm{~A}$ & $12,20 \mathrm{~A}$ & $128,00 \mathrm{~A}$ \\
\hline $\mathrm{CV} \%$ & 6,40 & 7,83 & 13,35 & 15,83 \\
\hline & \multicolumn{4}{|c|}{ 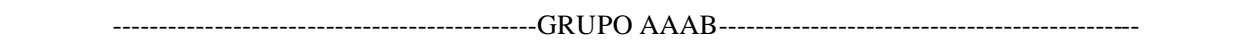 } \\
\hline 'Fhia 01' & $3,38 \mathrm{~A}$ & $101,80 \mathrm{~A}$ & $11,90 \mathrm{~A}$ & $146,60 \mathrm{~A}$ \\
\hline 'Prata Graúda' & $3,48 \mathrm{~A}$ & $94,00 \mathrm{AB}$ & $10,40 \mathrm{~A}$ & $153,80 \mathrm{~A}$ \\
\hline 'Fhia 18' & $3,52 \mathrm{~A}$ & $93,40 \mathrm{AB}$ & $11,10 \mathrm{~A}$ & $163,40 \mathrm{~A}$ \\
\hline 'Maçã Tropical' & $3,62 \mathrm{~A}$ & $88,60 \mathrm{~B}$ & $10,40 \mathrm{~A}$ & $90,00 \mathrm{~B}$ \\
\hline $\mathrm{CV} \%$ & 4,24 & 5,44 & 9,63 & 15,13 \\
\hline
\end{tabular}

Médias seguidas das mesmas letras na coluna não diferem entre si pelo teste de Tukey a 5\% de probabilidade.

ao passo que, nos grupos genômicos $\mathrm{AAB}$ e $\mathrm{AAAB}$, 'Prata Zulu' e 'Fhia 01' se destacaram com circunferência de 84,80 e $101,80 \mathrm{~cm}$, respectivamente.

O diâmetro do pseudocaule é importante no melhoramento genético da bananeira, pois está relacionado ao vigor, e reflete a capacidade de sustentação do cacho. Segundo SILVA (1999), os genótipos que apresentam maior diâmetro do pseudocaule são menos suscetíveis ao tombamento.

As médias referentes ao número de folhas não variaram entre os genótipos de nenhum grupo genômico. Porém, todos os acessos apresentaram um número acima de oito folhas por planta. Segundo SOTO BALLESTERO (1992), a presença de mais de oito folhas no florescimento é um fator considerado como suficiente para o desenvolvimento normal do cacho, indicando que os genótipos avaliados apresentaram cachos bem desenvolvidos.

Neste trabalho foram constatadas diferenças significativas para todos os grupos genômicos em relação ao número de dias do florescimento à colheita. No grupo genômico AAA, os genótipos que apresentaram menor número de dias do florescimento à colheita foram 'Nanicão' e 'Nam', com 103 e 100 dias, respectivamente. Todavia, 'Thap Maeo' (AAB) e 'Maçã Tropical' (AAAB) tiveram menor ciclo, com 96 e 90 dias, respectivamente. Os dados deste trabalho estão de acordo com os encontrados por
RAMOS et al. (2009), que, trabalhando com os mesmos genótipos, observaram que 'Nam', 'Thap Maeo' e 'Maçã Tropical' apresentaram menor ciclo do florescimento à colheita em relação aos demais cultivares pertencentes a cada grupo genômico.

Entretanto, PEREIRA et al. (2003), em LavrasMG, relataram que 'Nam' apresentou 185 dias da floração à colheita, apresentando um maior ciclo em relação a este trabalho. Dependendo da região de cultivo, pode ocorrer variação nos resultados referentes ao ciclo da planta. Para GOMES (1980), essa variação pode ser devido à altitude e ao clima, pois estes dois fatores exercem influência considerável sobre o fenótipo das plantas.

Os dados de produção são apresentados nas tabelas 02 e 03. No grupo AAA, 'Grand Naine', e 'Fhia 01 ', pertencente ao grupo AAAB, apresentaram maiores valores para peso dos frutos, peso do engaço, peso do cacho e, consequentemente, maior produtividade. Em relação ao grupo $\mathrm{AAB}$, houve diferença apenas para peso do engaço e número de frutos por cacho, com médias semelhantes para os demais atributos avaliados, o que demonstra que, para a região em que o experimento foi executado, os três cultivares deste grupo apresentaram praticamente o mesmo potencial produtivo.

O número de frutos, segundo SILVA et al. (1999), é fundamental na determinação do tamanho e 
Tabela 2 - Valores médios de peso de frutos (PF), peso do engaço (PENG), peso do cacho (PC), número de frutos por cacho $\left(\mathrm{N}^{\circ} \mathrm{F}\right)$, peso médio dos frutos (PMF) e produtividade (PROD) de acessos de bananeira em Botucatu-SP (março de 2009).

\begin{tabular}{|c|c|c|c|c|c|c|}
\hline Cultivar & $\mathrm{PF}(\mathrm{kg})$ & PENG (kg) & $\mathrm{PC}(\mathrm{kg})$ & № $\mathrm{F}$ & PMF (g) & PROD $\left(\mathrm{t} \mathrm{ha}^{-1}\right)$ \\
\hline & \multicolumn{6}{|c|}{ - } \\
\hline 'Grand Naine' & $28,42 \mathrm{~A}$ & $2,20 \mathrm{~A}$ & $30,34 \mathrm{~A}$ & $167 \mathrm{AB}$ & $169,62 \mathrm{~A}$ & $45,47 \mathrm{~A}$ \\
\hline 'Nanicão' & $19,27 \mathrm{~B}$ & $1,76 \mathrm{AB}$ & $21,03 \mathrm{~B}$ & $148 \mathrm{~B}$ & $130,33 \mathrm{~A}$ & $30,83 \mathrm{~B}$ \\
\hline 'Caipira’ & $14,48 \mathrm{BC}$ & $1,38 \mathrm{~B}$ & $15,86 \mathrm{BC}$ & $194 \mathrm{~A}$ & $76,03 \mathrm{~B}$ & $23,17 \mathrm{BC}$ \\
\hline 'Nam' & $10,32 \mathrm{C}$ & $1,33 \mathrm{~B}$ & $11,65 \mathrm{C}$ & $134 \mathrm{~B}$ & $76,98 \mathrm{~B}$ & $16,51 \mathrm{C}$ \\
\hline \multirow[t]{2}{*}{$\mathrm{CV} \%$} & 23,37 & 20,18 & 23,08 & 15,25 & 20,41 & 23,38 \\
\hline & & \multicolumn{2}{|c|}{--------GRUPO AAB----- } & & --------- \\
\hline 'Thap Maeo' & $17,72 \mathrm{~A}$ & $1,06 \mathrm{~B}$ & $19,14 \mathrm{~A}$ & $178 \mathrm{~A}$ & $98,13 \mathrm{~A}$ & $28,34 \mathrm{~A}$ \\
\hline 'Prata Anã' & $14,45 \mathrm{~A}$ & $1,24 \mathrm{AB}$ & $15,73 \mathrm{~A}$ & $132 \mathrm{~B}$ & $110,35 \mathrm{~A}$ & $23,13 \mathrm{~A}$ \\
\hline 'Prata Zulu' & $16,71 \mathrm{~A}$ & $1,74 \mathrm{~A}$ & $18,45 \mathrm{~A}$ & $153 \mathrm{AB}$ & $109,17 \mathrm{~A}$ & $26,73 \mathrm{~A}$ \\
\hline \multirow[t]{2}{*}{$\mathrm{CV} \%$} & 17,53 & 22,31 & 16,52 & 13,24 & 19,9 & 17,53 \\
\hline & \multicolumn{2}{|l|}{-} & \multicolumn{2}{|c|}{----GRUPO AAAB--- } & $190,21 \mathrm{~A}$ & 52,58 A \\
\hline 'Prata Graúda' & $24,33 \mathrm{~B}$ & $2,30 \mathrm{~B}$ & $26,63 \mathrm{~B}$ & $148 \mathrm{~B}$ & $165,65 \mathrm{~A}$ & $38,93 \mathrm{~B}$ \\
\hline 'Fhia 18' & $19,80 \mathrm{~B}$ & $2,26 \mathrm{~B}$ & $22,00 \mathrm{~B}$ & $184 \mathrm{~A}$ & $100,02 \mathrm{~B}$ & $31,68 \mathrm{~B}$ \\
\hline 'Maçã Tropical' & $9,89 \mathrm{C}$ & $1,61 \mathrm{C}$ & $11,49 \mathrm{C}$ & $123 \mathrm{C}$ & 80,82 B & $14,96 \mathrm{C}$ \\
\hline $\mathrm{CV} \%$ & 16,77 & 15,43 & 16,16 & 7,49 & 15,51 & 17,27 \\
\hline
\end{tabular}

Médias seguidas das mesmas letras na coluna não diferem entre si pelo teste de Tukey a 5\% de probabilidade.

peso do cacho, revelando importância no melhoramento genético, estando diretamente relacionado com o número de pencas.

RAMOS et al. (2009), trabalhando com os mesmos genótipos, constataram maiores valores em relação ao peso dos frutos, peso do engaço, peso do cacho e produtividade para 'Grande Naine' e 'Nanicão'
(AAA), 'Prata-Zulu' e 'Thap Maeo' (AAB), 'FHIA 01' e 'Prata-Graúda' (AAAB). DAMATTO JÚNIOR et al. (2005), em Botucatu-SP, citaram que a bananeira'PrataZulu' apresentou cachos pesando $33,9 \mathrm{~kg}$ e produtividade de $54,3 \mathrm{tha}^{-1}$.

Segundo SILVA et al. (2006), o atributo número de pencas possui grande interesse para o

Tabela 3 - Valores médios de número de pencas $\left(\mathrm{N} N^{\mathrm{o}} \mathrm{P}\right)$, peso da $2^{\underline{a}}$ penca $\left(\mathrm{P} 2^{\underline{a}} \mathrm{P}\right)$, número de frutos na $2^{\underline{\underline{a}}}$ penca $\left(\mathrm{F} 2^{\underline{\underline{a}}} \mathrm{P}\right)$, comprimento $(\mathrm{CF}) \mathrm{e}$ diâmetro de frutos (DF) de acessos de bananeiras, Botucatu-SP (março 2009).

\begin{tabular}{|c|c|c|c|c|c|}
\hline Cultivar & № $\mathrm{P}$ & $\mathrm{P} 2^{\mathrm{a}} \mathrm{P}(\mathrm{kg})$ & $\mathrm{F} 2^{2} \mathrm{P}$ & $\mathrm{CF}$ & DF \\
\hline & \multicolumn{5}{|c|}{ 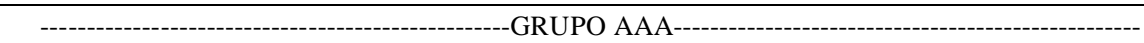 } \\
\hline 'Grand Naine' & $9,60 \mathrm{AB}$ & $3,84 \mathrm{~A}$ & $19,80 \mathrm{AB}$ & $22,93 \mathrm{~A}$ & $36,92 \mathrm{~A}$ \\
\hline 'Nanicão’ & $10,20 \mathrm{~A}$ & $3,20 \mathrm{AB}$ & $19,00 \mathrm{~B}$ & $21,30 \mathrm{~A}$ & $37,74 \mathrm{~A}$ \\
\hline 'Nam’ & $9,80 \mathrm{~A}$ & $2,06 \mathrm{BC}$ & $26,00 \mathrm{~A}$ & $15,89 \mathrm{~B}$ & $30,52 \mathrm{~B}$ \\
\hline 'Caipira' & $8,20 \mathrm{~B}$ & $1,63 \mathrm{C}$ & $19,40 \mathrm{~B}$ & $15,40 \mathrm{~B}$ & $31,80 \mathrm{~B}$ \\
\hline $\mathrm{CV} \%$ & 10,23 & 23,89 & 16,8 & 7,05 & 5,94 \\
\hline 'Thap Maeo' & 1202 A & $1844_{1}$ & GRUPO A & $1537 \mathrm{~B}$ & ------- \\
\hline 'Prata Anã' & $9,60 \mathrm{~B}$ & $\begin{array}{l}1,04 \mathrm{~A} \\
1,81 \mathrm{~A}\end{array}$ & $\begin{array}{l}21,00 \mathrm{~A} \\
16,00 \mathrm{~B}\end{array}$ & $17,96 \mathrm{~A}$ & $\begin{array}{l}5,00 \mathrm{~A} \\
36,16 \mathrm{~A}\end{array}$ \\
\hline 'Prata Zulu' & $9,80 \mathrm{~B}$ & $1,80 \mathrm{~A}$ & $15,00 \mathrm{~B}$ & $16,78 \mathrm{AB}$ & $36,00 \mathrm{~A}$ \\
\hline $\mathrm{CV} \%$ & 9,55 & 18,61 & 13,08 & 7,44 & 3,99 \\
\hline & & & RUPO AA & 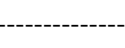 & - \\
\hline 'Fhia 01' & $11,10 \mathrm{~A}$ & $3,25 \mathrm{~A}$ & $18,00 \mathrm{~A}$ & $24,30 \mathrm{~A}$ & $39,36 \mathrm{~A}$ \\
\hline 'Prata Graúda' & $11,03 \mathrm{~A}$ & $3,15 \mathrm{~A}$ & $18,00 \mathrm{~A}$ & $22,06 \mathrm{~A}$ & $39,76 \mathrm{~A}$ \\
\hline 'Fhia 18' & $9,40 \mathrm{~B}$ & $2,29 \mathrm{AB}$ & $17,00 \mathrm{~A}$ & $18,52 \mathrm{~B}$ & $34,27 \mathrm{~B}$ \\
\hline 'Maçã Tropical' & $8,20 \mathrm{~B}$ & $1,62 \mathrm{~B}$ & $17,00 \mathrm{~A}$ & $16,20 \mathrm{~B}$ & $31,98 \mathrm{C}$ \\
\hline $\mathrm{CV} \%$ & 8,75 & 23,65 & 5,69 & 6,57 & 3,43 \\
\hline
\end{tabular}

Médias seguidas das mesmas letras na coluna não diferem entre si pelo teste de Tukey a 5\% de probabilidade.

Ciência Rural, v.41, n.4, abr, 2011. 
produtor, assim como para o melhoramento genético da bananeira, uma vez que se constitui na unidade comercial. A característica massa da segunda penca é utilizada como padrão para a análise de uma série de atributos, como: determinação do ponto de colheita, peso médio do fruto, comprimento e diâmetro do fruto, além do teor de sólidos solúveis, açúcar e acidez (JARAMILLO, 1982; SOTO BALLESTERO, 1992).

'Grand Naine' foi o cultivar em destaque do grupo genômico AAA, com valores maiores para peso da 2a penca, não diferindo de 'Nanicão' e 'Nam' em número de pencas por cacho e comprimento e diâmetro da 2a penca. 'Fhia 01' e 'Prata Graúda' (AAAB) apresentaram maiores médias para números de pencas por cacho, peso, comprimento e diâmetro dos frutos da 2 a penca. Referente ao grupo AAB, Thap Maeo foi o melhor cultivar em relação ao número de pencas por cacho e número de frutos na $2^{2}$ penca, porém não diferiu dos demais cultivares no peso e diâmetro dos frutos da 2 a penca.

RAMOS et al. (2009), avaliando diferentes genótipos de bananeira, em Botucatu-SP, observaram que 'Grand Naine' apresentou maior peso da 2 a penca quando comparado aos cultivares 'Nanicão', 'Nam' e 'Caipira', corroborando com os valores observados neste trabalho. Em Lavras-MG, PEREIRA et al. (2003), verificaram que os frutos dos híbridos 'Fhia 01', 'Prata Graúda' e 'Fhia 18' atingiram comprimentos de 16,22, 17,84 e 14,46 cm, respectivamente.

\section{CONCLUSÃO}

Os genótipos que apresentaram melhor desempenho agronômico dentro de cada grupo foram 'Grand Naine'(AAA), 'Thap Maeo' (AAB) e 'Fhia 01' (AAAB).

\section{REFERÊNCIAS}

ANUÁRIO BRASILEIRO DA FRUTICULTURA. Santa Cruz do Sul: Gazeta Santa Cruz, 2009. 136p.

CUNHA, A.R. et al. Classificação climática para o município de Botucatu, SP, segundo Köppen. In: SIMPÓSIO EM ENERGIA NA AGRICULTURA, 1999, Botucatu, SP. Anais... Botucatu: Faculdade de Ciências Agronômicas, 1999. p.490-491.

DAMATTO JUNIOR, E.R. et al. Produção e caracterização de frutos de bananeira 'Prata-Anã' e 'Prata Zulu'. Revista Brasileira de Fruticultura, v.27, n.3, p.440-443, 2005. Disponível em: <http://www.scielo.br/
scielo.php?script=sci_arttext\&pid=S01002945200500030024\&lng= pt\&nrm=iso\&tlng=pt $>$. Acesso em: 14 dez. 2010. doi: $10.1590 / \mathrm{S} 010029452005000300024$.

EMBRAPA. Centro Nacional de Pesquisa de Solos. Sistema Brasileiro de Classificação de Solos. 2.ed. Rio de Janeiro: EMBRAPA SOLOS, 2006. 306p.

GOMES, W.R. Exigências climáticas da cultura da bananeira. Informe Agropecuário,v.6, n.63, p.14-15, 1980.

GONÇALVES, V.D. et al. Avaliação dos cultivares de bananeira 'PrataAnã', 'Thap Maeo' e 'Caipira' em diferentes sistemas de plantio no norte de minas gerais. Revista Brasileira de Fruticultura, v.30, n.2, p.371-376, 2008. Disponível em: <http://www.scielo.br/ scielo.php?script=sci_arttext\&pid=S010029452008000200018\&lng $=$ pt\&nrm=iso\&tlng=pt $>$. Acesso em: 17 dez. 2010. doi: 10.1590/ S0100-29452008000200018.

JARAMILLO, R.C. Las principales características morfológicas del fruto de banano, variedade Cavendish Gigante (Musa AAA) em Costa Rica. Upeb: Impretex,1982. $42 \mathrm{p}$.

LEDO, A.S. et al. Avaliação preliminar de genótipos de banana (Musa spp.) em Rio Branco (AC). Revista Brasileira de Fruticultura, v.19, n.1, p.51-56, 1997.

LEDO, A.S. et al. Avaliação de genótipos de bananeira na região do Baixo São Francisco, Sergipe. Revista Brasileira de Fruticultura, v.30, n.3, p.691-695, 2008. Disponível em: <http://www.scielo.br/ scielo.php?script=sci_arttext\&pid=S010029452008000300022\&ln $\mathrm{g}=\mathrm{pt} \& \mathrm{nrm}=\mathrm{iso} \& t \operatorname{lng}=\mathrm{pt}>$. Acesso em: $15 \mathrm{dez} .2010$. doi: 10.1590/ S0100-29452008000300022.

PEREIRA, L.V. et al. Avaliação de cultivares e híbridos de bananeira em Lavras, MG. Ciência e Agrotecnologia, v.27, n.1, p.17-25, 2003.

RAMOS, D.P. et al. Avaliação de genótipos de bananeira em Botucatu-SP. Revista Brasileira de Fruticultura, v.31, n.4, p.1092-1101, 2009. Disponível em: <http://www.scielo.br/ scielo.php?script=sci_arttext $\&$ pid $=$ S010029452009000400024\&ln $\mathrm{g}=\mathrm{pt} \& \mathrm{nrm}=\mathrm{iso} \& \mathrm{t} \operatorname{lng}=\mathrm{pt}>$. Acesso em: $15 \mathrm{dez}$. 2010. doi: 10.1590/S0100-29452009000400024.

SILVA, S.O. et al. Cultivares. In: ALVES, E.J. (Org.). A cultura da banana: aspectos técnicos, socieconômicos e agroindustriais. 2.ed. rev. Brasília: Embrapa - SPI/ Embrapa-CNPMF, 1999. p. $85-105$.

SILVA, S. de O. et al. Avaliação de clones de banana Cavendish. Ciência e Agrotecnologia, v.30, p.832-837, 2006.

SOTO BALLESTERO, M. Banana: cultivo e comercialización. San José: Litografia y Imprensa, 1992. 674p.

TEIXEIRA, L.A.J. et al. Banana. Boletim Técnico do Instituto Agronômico de Campinas, n.100, p.131-132, 1997. 